\title{
Preface of the special issue for Gheorghe Păun's 70th anniversary
}

\author{
Marian Gheorghe $^{1} \cdot$ Mario J. Pérez-Jiménez ${ }^{2} \cdot$ Grzegorz Rozenberg $^{3,4} \cdot$ Arto Salomaa $^{5}$
}

Published online: 3 December 2020

(c) Springer Nature Singapore Pte Ltd. 2020

This special issue of the Journal of Membrane Computing is honouring Gheorghe Păun's landmarking contributions to the creation and further development of Membrane Computing, on the occasion of his 70th birthday.

Membrane Computing is a research area of natural computing initiated by "Computing with Membranes" paper, published in Journal of Computer and System Sciences, 61, 1 (2000) 108-143, first circulated as TUCS report no. 208, 1998, University of Turku, Finland.

The field of Membrane Computing has witnessed, immediately after its launch, a vigorous development that has continued over the years until now. The large and impressive literature of this area consists of contributions of a theoretical nature rooted in computer science and mathematics (often inspired by concepts and phenomena from molecular and cell biology), as well as applications to modelling problems in areas, such as, biology, distributed computing, graphics, optimisations and evolutionary computing, systems and synthetic biology, ecology, and robotics.

In 2010, a handbook presenting the state-of-the-art results at that time, edited by Gh. Păun, G. Rozenberg and A. Salomaa, was published by Oxford University Press. Many other volumes, investigating various facets of the Membrane Computing research, have been published. The models of this

Mario J. Pérez-Jiménez

marper@us.es

Marian Gheorghe

M.Gheorghe@bradford.ac.uk

Grzegorz Rozenberg

grozenberg@gmail.com

Arto Salomaa

asalomaa@utu.fi

1 University of Bradford, Bradford, UK

2 University of Sevilla, Seville, Spain

3 Leiden University, Leiden, The Netherlands

4 University of Colorado at Boulder, Boulder, CO, USA

5 University of Turku, Turku, Finland computing paradigm have been called membrane systems or $\mathrm{P}$ systems ( $\mathrm{P}$, coming from Păun).

Gheorghe Păun had a significant impact on a number of scientific disciplines, such as formal language theory and automata, regulated rewriting, cooperating distributed grammar systems, DNA computing, and theoretical foundation of linguistics.

He is also deeply involved in the Romanian cultural life. For example, he is the founder and editor-in-chief of the cultural magazine "Curtea de la Argeş" (published since December 2010), an influential voice in the Romanian cultural environment.

Gheorghe Păun has published more than 500 scientific publications (many of them in collaboration with more than 100 researchers) and over 50 volumes, including books on games and literary books such as science fiction, novels, poetry, essays, and memoirs. He also edited more than 100 scientific and cultural volumes. Numerous of his books have been translated in many languages, including Japanese, Chinese, Russian, English, Hungarian, and Italian. Gheorghe Păun is also the honorary editor-in-chief of the Journal of Membrane Computing. His enormous contribution has been recognised by the scientific community: among others, he is an ISI "highly cited researcher" and holder of five Doctor Honoris Causa honorary degrees.

The editors would like to thank all the contributors, as well as the reviewers, for their effort in producing this special issue.

- The paper "Computing with SN P systems with I/O mode", by Henry N. Adorna, deals with SN P systems simulating uniform sequential computing models. SN P modules are described, differentiated from SN P systems by the input/output nature. It is demonstrated that any finite automaton can be simulated by some SN P module. The idea of $\mathrm{P}$ automatic sequences is introduced, demonstrating some of their properties.

- The paper "Reversible Computation in Nature Inspired Rule-Based Systems", by Bogdan Aman and Gabriel Ciobanu, studies the reversibility of rule-based sys- 
tems existing in the area of natural computing. Different properties of reversible systems are demonstrated throughout the work, such as the applicability of the rules, diamond properties; that is, the commutative application of two different sets of rules, and loop properties; that is, the cancellation between two different sets of rules leading to the initial configuration.

- In the paper "Sequential dynamic threshold neural $\mathrm{P}$ systems", the authors Tingting Bao, Nan Zhou, Zeqiong Lv, Hong Peng and Jun Wang explore the computational power of sequential dynamic threshold neural $\mathrm{P}$ systems. Apart from typical neurons, these systems have threshold units capable of enabling or disabling certain rules, depending on the number of spikes remaning in them. The Turing completeness of this model with four different sequentiality strategies is proven.

- The paper "P Colonies and Reaction Systems", by Lucie Ciencialová, Luděk Cienciala and Erzsébet Csuhaj-Varjú introduces a new model of $\mathrm{P}$ colonies where environment is represented as a set, being used to simulate interactive processes in a given reaction system. Each computational step of the reaction system by four steps of the P colony, in such a way that objects are generated and multiplied to let them be accesible for every agent simulating the enabled reaction, then the reaction is simulated and finally the unused objects are consumed.

- Thomas Hinze describes in the paper "Coping with Dynamical Reaction System Topologies using Deterministic P Modules: A Case Study of Photosynthesis" a way to simulate the triggers that enable chemical reactions in real-life processes. This is done by means of deterministic $\mathrm{P}$ modules, indicating a list of input/output signal identifiers that will be transformed within these modules. Photosynthesis is used as an example for the process of the plants absorbing light energy to produce fructose.

- Sergiu Ivanov and Ion Petre introduce in their paper "Controllability of reaction systems" the concept of controllability of reaction systems as the ability of transitioning between any two states through a suitable choice of context sequence. The problem is demonstrated to be PSPACE-complete. Besides, a model of oncogenic signalling based on reaction systems is used to illustrate the intricacies of the controllability of reaction systems.

- The paper "Simulating counting oracles with cooperation", by Alberto Leporati, Luca Manzoni, Giancarlo Mauri, Antonio E. Porreca and Claudio Zandron gives a proof of the characterisation of monodirectional shallow chargeless $\mathrm{P}$ systems with active membranes and minimal cooperation working in polynomial time by $\mathbf{P}^{\# \mathbf{P}}$. This is done, first, by means of simulating a single \#P query with this type of membrane systems, and second, by simulating these $\mathrm{P}$ systems with a single \#P query.
- In the paper "Solving a PSPACE-complete Problem with cP Systems", by Radu Nicolescu, Michael Dinneen and Alec Henderson, an efficient deterministic solution to QSAT is given by means of $\mathrm{P}$ systems with complex objects. The solution only uses a small amount of custom alphabet symbols (19), a small constant number of rules (10), and a small constant upper limit of membrane nesting depth (6), independent of the problem size.

- The paper "Seeking computational efficiency boundaries: The Păun's conjecture”, by David Orellana-Martín and Agustín Riscos-Núñez is a recopilarory of results concerning the Păun's conjecture and some frontiers of efficiency found in the framework of Membrane Computing. As stated in the manuscript, some partial solutions to the conjecture have been found through different lines of research, and different approaches to similar classes of membrane systems have been studied.

- The paper "The computational power of cell-like P systems with one protein on membrane", by Bosheng Song, Xiaoyan Luo, Luis Valencia-Cabrera and Xiangxiang Zeng, explores the role of the proteins in the path to achieve Turing completeness in membrane systems. In this work, the authors demonstrate that a single protein that can change by the application of rules is enough for a P system with two membranes; that is, only one level of depth.

- The paper "Description of Membrane Systems with Time Petri Nets: Promoters/Inhibitors, Membrane Dissolution, and Priorities", by György Vaszil and Péter Battyányi describes relations between Petri nets and different types of membrane systems. This research line is extended in such a way that membrane systems with promoters/inhibitors, membrane dissolution and priority of the rules can be simulated with time Petri nets while preserving the restriction of the non-determinism of the firings of the transitions.

- The paper "A Formal Framework for Spiking Neural P systems", by Sergey Verlan, Rudolf Freund, Artiom Alhazov, Sergiu Ivanov and Linqiang Pan presents an extension of a formal framework for P systems, where spiking neural $\mathrm{P}$ systems are also captured. The concepts of input and output are not usual outside of the domain SN P systems, thus it is introduced to formalise them in the general formal framework. Different extensions to SN $P$ systems, such as extended rules, astrocytes and derivation modes are introduced in the formal framework.

- The paper "Generating Pictures in String Representations with P Systems: The Case of Space-Filling Curves", by Rodica Ceterchi and K. G. Subramanian, continues the research line joining space filling curves and $\mathrm{P}$ systems. More precisely, the idea is to generate the former by means of rules from $\mathrm{P}$ systems. In the manuscript, $\mathrm{P}$ systems generating chain codes for Peano, Wunderlich and 
Hilbert words are introduced. Besides, they demonstrate that there exist $\mathrm{P}$ systems generating the approximation polygons of Hilbert, Peano and Lebesgue curves. The representation in two dimensions of the Peano curve is developed by means of parallel array rewriting rules with membrane systems.

- The paper "From P Systems to Morphogenetic Systems: an Overview and Open Problems", by Petr Sosík, Jan Drastík, Vladimír Smolka and Max Garzon discuses about the relations between morphogenetic (M) systems and and $\mathrm{P}$ systems. $\mathrm{M}$ systems have been demonstrated to be universal systems. Besides, a polynomial-time solution to 3-SAT is recalled. Some open problems and experiments with $\mathrm{M}$ systems are commented, such as the simulation of a septum division process in prokayotic cells and the simulation of the growth and division of eukaryotic cells with nucleusand cytoskeleton.

- The paper Simulation Challenges in Membrane Computing”, by Luis Valencia-Cabrera, Ignacio Pérez-Hurtado and Miguel Á. Martínez-del-Amor talks about the different problems found in the implementation of simulators of $\mathrm{P}$ systems. Used as assistants for the researchers in the area, simulators must be suitable to deal with large amounts of data. Therefore, an efficient implementation is necessary. Besides, graphical user interfaces are critical to let non-experts on Membrane Computing to control the implemented P systems.

We offer this special issue to Gheorghe Păun, as a token of appreciation for his outstanding scientific contributions. We thank Gheorghe Păun, also on behalf of the Membrane Computing community, for launching and tirelessly supporting this scientific area, as well as for being a mentor for many young researchers and for creating a large and thriving research community which continues to expand the research in Membrane Computing in many novel and interesting research directions.

Publisher's Note Springer Nature remains neutral with regard to jurisdictional claims in published maps and institutional affiliations. 